Research Article

\title{
Improving the Quality of Left-Behind Children's Participation in Sports through Wireless Network Monitoring
}

\author{
Jinjin Zhao iD \\ Jilin Agricultural University, Changchun 130118, China \\ Correspondence should be addressed to Jinjin Zhao; zhaojinjin@jlau.edu.cn
}

Received 30 July 2021; Accepted 24 August 2021; Published 8 September 2021

Academic Editor: $\mathrm{Ke} \mathrm{Gu}$

Copyright ( 92021 Jinjin Zhao. This is an open access article distributed under the Creative Commons Attribution License, which permits unrestricted use, distribution, and reproduction in any medium, provided the original work is properly cited.

Urbanization is the process that people shift from rural to urban areas, which has led to large numbers of left-behind children in China. The left-behind children stay in rural regions of China while their parents work in urban areas. The left-behind children have few opportunities to participate in sports due to the lacking of concern, and it is not of high quality even though they participate in sports. Therefore, it is necessary to improve the quality of left-behind children's sports participation through wireless network monitoring. Wireless network monitoring transmits high-definition (HD) video streaming in real time to facilitate feedback timely. This paper studies the two-dimensional (2D) integer discrete cosine transform (DCT) and analyzes the reason for image distortion, then an improved DCT coefficient quantization approach is proposed for long-distance real-time transmission of HD video streaming, and a noise processing with a zero-mean noise processing is added in optimized approach to solve the image distortion problem. The experimental results show that the proposed improved approach has a good performance in reducing the blocking artifacts, and within the image reconstruction, the proposed approach improves the subjective video quality.

\section{Introduction}

The problem of left-behind children in China has become prominent over the past decades. More and more young and middle-aged farmers have worked in urban areas with the rapid advance in China's economy, and large numbers of left-behind children have emerged in the rural areas. According to related surveys, there are more than 10 million left-behind children in rural areas in China. Left-behind children have attracted the attention of the whole society. At present, the problems of left-behind children in health and education are more prominent, and most of them lack physical exercise, so it is necessary to improve the quality of left-behind children's sports participation through wireless network monitoring [1].

Wireless network monitoring transmits high-definition video streaming in real time to facilitate feedback timely [2]. The HD video streaming consists of moving images by frame one after another $[3,4]$. When the images are continuously displayed at a certain rate, people will have a sense of video due to the persistence of vision by eyes. The processing of video streaming can be roughly divided into the following three steps: (i) the original HD video streaming information is processed digitally, including quantization, coding, and other processes; (ii) the digital HD video streaming is transmitted to the receiver; and (iii) receiver will receive digital HD video streaming for antidigital processing, including inverse quantization, decoding, and other processes. Video monitoring is completed by the above steps in order to observe the quality of left-behind children's participation in sports.

High-Definition Multimedia Interface (HDMI) is a digital video/audio interface technology for video transmission; at the same time, the receiver can receive audio signals [5]. HDMI transmission distance is up to 30 meters, so it is not suitable for long-distance transmission. The HDMI cable can be seen as a low-pass filter. If the digital signals of $\mathrm{HD}$ video streaming are transmitted over long distances through the related interface, the high-frequency components will inevitably be severely attenuated. In 
addition, the transmission rate of digital signals of HD video streaming is very fast and more likely to cause intersymbol interference, so that the receiver is difficult to decode and display. Therefore, in order to realize real-time and longdistance transmission of $\mathrm{HD}$ video streaming, it is necessary to compress the signal source. The improved DCT quantization approach can reserve the raw data to the greatest extent, which means eliminating redundant information as far as possible. As a result, it is necessary to compress the HD video streaming of HDMI.

Accordingly, the main contributions of this paper are summarized as follows: (i) an improved DCT coefficient quantization approach for long-distance real-time transmission of HD video streaming is proposed and (ii) a noise processing with a zero-mean noise processing is added in an optimized approach to solve the image distortion problem.

The remainder of this paper is organized as follows. Section 2 reviews the related work. In Section 3, the 2D DCT approach is studied. In Section 4, an improved DCT coefficient quantization approach is proposed. The experimental results are shown in Section 5. Section 6 concludes this paper.

\section{Related Work}

Many strategies of DCT for video or image processing have been proposed. In [6], a new multiobjective optimization algorithm was proposed to search for an efficient integer DCT matrix, which had the coding performance as close as possible to the transform in high-efficiency video coding but implemented with reduced hardware and power. In [7], an efficient hybrid image fusion method was proposed which is suitable for visual sensor networks based on the integer lifting wavelet transform and the DCT. In [8], a reconfigurable transform architecture was presented to flexibly support the reusability of different transform sizes. The proposed architecture maximally reused the hardware resources by rearranging the order of input data for different transform sizes while still exploiting the butterfly property. In [9], a new approximation for the 8-point discrete tchebichef transform was proposed with a higher power-and compression efficiency by exploring coefficient truncation. In [10], an image-dependent optimum nonnegative integer bit allocation algorithm was proposed, which was then mapped into desired image-independent solution via utilization of a prepared combined image and proposed modified step size mapping technique. In [11], the authors presented a hardware architecture for $8 \times 82 \mathrm{D}$ DCT and inverse DCT using Taylor-series expansion of trigonometric functions. In [12], the authors presented an efficient and low complexity integer approximation of the DCT for image compression. Their new approach involved replacing the bit shift elements of a variant of the signed DCT transform by zeros, in order to eliminate the bit shift operations. In [13], an efficient hardware implementation was proposed for high-speed vector-radix decimation-in-frequency three-dimensional DCT with an optimum area and power consumption. In [14], a novel algorithm was proposed to determine the minimum number of low-frequency DCT coefficients required for transform and quantization block in high-efficiency video coding. In [15], a novel semifragile watermarking technique using integer wavelet transform and DCT for tamper detection and recovery to enhance enterprise multimedia security was proposed. In [16], a new model of inverse DCT kernel for high-efficiency video coding was proposed. In [17], the authors presented a blind and robust scheme using $\mathrm{YCbCr}$ color space, integer wavelet transform, and DCT for color image watermarking.

Some studies on blocking artifacts have also been proposed. In [18], the authors presented a deep network to eliminate image compression artifacts (usually denoted by image deblocking) based on image fusion in a multiscale manner. In [19], a novel dual-residual network was proposed to reduce compression artifacts caused by lossy compression codecs. In [20], a new method of image upscaling along with deblocking of compressed images was proposed. In [21], a wavelet transform based on the Meyer algorithm with edgeangle tracking capability was proposed for edge and blocking artifact reduction of an image, during image compression processes. In [22], a blocking artifact detection method was proposed for motion-compensated frame-rate upconversion algorithms. In [23], the authors presented the design of a partial overlapping block using exact Legendre moment computation for gray-level image reconstruction. There have also been several researches with respect to image compression [24-27].

\section{Image Compression}

Improving the quality of left-behind children's participation in sports is mainly achieved through wireless network monitoring, and how to improve the quality of video has become the key to research. The large volume of HD video streaming is well beyond the Ethernet transmission ability, and there are strict requirements in the real-time performance of $\mathrm{HD}$ video, so it needs to be a more appropriate compression approach to perform the compression of realtime HD video streaming. Thus, the real-time transmission of peer to peer can be realized by using HDMI through Ethernet. Theoretically, HDMI can send both video and audio streaming at a data transfer rate up to $4.5 \mathrm{~Gb} / \mathrm{s}$. According to the above, taking the resolution of $1920 \times 1080$ $\mathrm{HD}$ video streaming as an example, which requires that the transfer rate must be $2 \mathrm{~Gb} / \mathrm{s}$. Considering the fact that no IP packet loss occurs when HD video streaming is transmitted over $100 \mathrm{M}$-Ethernet, the transfer rate on the physical link should be less than $100 \mathrm{Mb} / \mathrm{s}$. Consequently, the compression ratio should be $25: 1$ to $34: 1$.

3.1. DCT. The original HD video streaming is highly correlated, which also has enough redundancy, while using the compression approach is to eliminate redundancy and reserve the original information as much as possible. That is, by reducing the correlation of the $\mathrm{HD}$ video streaming sequence, less bits are used to quantify the $H D$ video streaming in order to compress the $\mathrm{HD}$ video streaming. There are three aspects in information redundancy that are 
interframe redundancy, intraframe redundancy, and information entropy redundancy.

Under such context, this paper has relatively strict requirements on real-time and compression complexity.
Accordingly, I use a DCT-based video compression algorithm while 2D DCT is defined as follows:

$$
\begin{gathered}
F(u, v)=\frac{2}{\sqrt{A B}} c(u) c(v)\left[\sum_{i=0}^{A-1} \sum_{j=0}^{B-1} f(i, j) \cos \frac{(i+0.5) u \pi}{A} \cos \frac{(j+0.5) v \pi}{B}\right], \\
F(i, j)=\frac{2}{\sqrt{A B}}\left[\sum_{i=0}^{A-1} \sum_{j=0}^{B-1} c(u) c(v) F(u, v) \cos \frac{(i+0.5) u \pi}{A} \cos \frac{(j+0.5) v \pi}{B}\right], \\
F=\left(A_{\text {int }} f A_{\text {int }}^{T}\right) \otimes E,
\end{gathered}
$$

where $u=0$ and $v=0$, and $c(u)=c(v)=(1 / \sqrt{2})$; when $u, v \neq 0, c(u)=c(v)=1$.

3.2. $2 D$ DCT Approach. The characteristics of human vision are not sensitive to the distortion of the image caused by the loss of high-frequency component packets in HD video streaming. In addition, there are a small number of high-frequency components in the image. Hence, the distributed energy in the image can be concentrated in the middle and low-frequency region by DCT. In other words, it makes the most of the energy of the image in the upper left of the DCT coefficients, and this can be transmitted to the receiver using a wireless network. Then, the receiver will realize the inverse transformation of the energy concentrated in the low-frequency parts of DCT so as to recover the original image information. However, from the DCT transformation formula, it can be seen that the computation of float is still huge, which conflicts with the requirements of low latency and low complexity in this paper; moreover, in the receiver decoding process, it will inevitably affect the accuracy. For this reason, the $2 \mathrm{D}$ integer DCT is used as the intraframe compression algorithm in this paper.

A 2D DCT can be realized by conducting one-dimensional DCT (1D DCT) twice, in which a 1D DCT is performed in the row first and then performed in the column. The 2D DCT can be represented by the matrix as shown in equation (2). Figuratively speaking, it is to figure out which $2 \mathrm{D}$ cosine waves constitute the image.

$$
F=A f A^{T},
$$

where $F$ is the coefficients of the transformation, $f$ is the pixels of the image, and $A$ is the transformation matrix.

In the process of $2 \mathrm{D}$ integer $\mathrm{DCT}$, in order to reduce the computation and complexity, all the elements of the transformation matrix $A$ are modified to integers and the scale factor matrix is added in the quantization process so as to realize the transformation and quantization. Meanwhile, the coding rate is also greatly improved. $2 \mathrm{D}$ integer DCT transformation matrix is defined as follows: where $E$ is the scale factor and $A_{\text {int }}$ is the transformation matrix.

In the video compression algorithm, the single frame from the video is composed of many $8 \times 8$-pixel blocks. To realize the domain transformation of the image content, $2 \mathrm{D}$ integer DCT is used to transform the $8 \times 8$-pixel blocks, respectively, and then 64 DCT coefficients can be obtained. This paper uses the $2 \mathrm{D}$ integer DCT to effectively avoid the error of precision in the computation of float in inverse DCT. The computation is greatly reduced to addition and subtraction and shift operation by means of $2 \mathrm{D}$ integer DCT, and the division is further avoided. Moreover, the data within the pixel block is highly correlated. In this way, a large number of 0 coefficients will appear in the lower right after 2D integer DCT, and then " $z$ " font will be used for scanning and coding, so that the volume of data will be much smaller. This lays a foundation for the realization of real-time transmission with low latency.

After using the above transformation processing, the next step is to use the conventional quantization method to operate the 64 DCT coefficients of each pixel block obtained by transformation. Quantization can not only adjust the low energy coefficient to zero which realizes certain compression of the image content but also reduce the transformed DCT coefficients, which provides convenience for the subsequent $8 \mathrm{~b} / 10 \mathrm{~b}$ code transmission. According to the characteristics of human vision, they are not sensitive to the loss of high-frequency components, so high-frequency information can be lost to compress large amounts of data. However, the adjacent pixel blocks $(8 \times 8)$ in the content of a single frame image are quantized separately, resulting in no relation between the quantization errors of adjacent blocks. For the boundary pixels of adjacent blocks, if the quantization error between adjacent blocks has a jump, the smooth texture in the original image content will change greatly on the boundary region between adjacent blocks, so that the image distortion gets more serious. I take a frame of left-behind children participating in sports from the video for analysis. The comparison of the $2 \mathrm{D}$ integer DCT compression effects is shown in Figure 1, and the comparison of local magnification of $2 \mathrm{D}$ integer DCT compression effects is shown in Figure 2. 

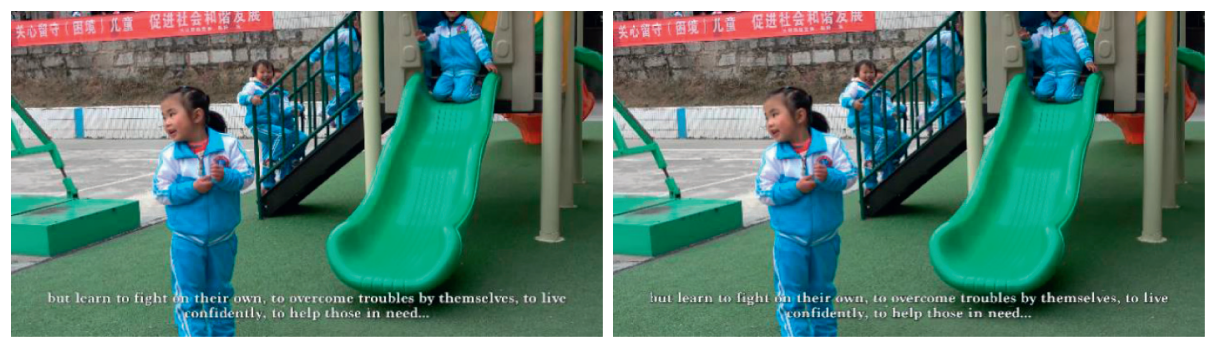

FIGURE 1: The comparison of the 2D integer DCT compression effects.

It can be seen from Figure 2 that the visual effect of local magnification of reconstructed images is obviously worse because there is a contradiction between the extremely limited bandwidth and the real-time transmission of big data. Therefore, it is necessary to compress the data source of $\mathrm{HD}$ video streaming effectively to realize the transmission of $\mathrm{HD}$ video streaming with low latency. And in the process of image domain transformation, DCT also greatly destroys the correlation between pixel blocks. In addition, the conventional quantization processing of transformed DCT coefficients will cause serious distortion to the reconstructed image at the receiver.

\section{Improved Approach}

4.1. The Reason for Image Distortion. In this paper, the $2 \mathrm{D}$ integer DCT is used as the intraframe compression algorithm, but the distortion of the image reconstructed by the receiver is still serious which is caused by the coefficient quantization in 2D integer DCT. The coefficient quantization process and reconstruction process of classical integer DCT are defined as follows:

$$
\begin{gathered}
F_{Q s}(u, v)=\operatorname{round}\left(\frac{F_{D}(u, v)}{Q s(u, v)}\right), \\
F_{R}(u, v)=F_{Q s}(u, v) Q s(u, v),
\end{gathered}
$$

where $F_{Q s}(u, v)$ is the $2 \mathrm{D}$ integer DCT coefficients after quantization, $F_{D}(u, v)$ is the $2 \mathrm{D}$ integer DCT coefficients before quantization, $Q s(u, v)$ is the quantization step size of the quantization process, $F_{R}(u, v)$ is the reconstructed $2 \mathrm{D}$ integer DCT coefficients, and round is the round function. Then the quantization error of the 2D integer DCT coefficients is defined as follows:

$$
\sigma(u, v)=F_{R}(u, v)-F_{D}(u, v) .
$$

There is still a strong correlation between adjacent pixel blocks in the original single frame image content before $2 \mathrm{D}$ integer DCT is used, but the DCT process broke the correlation between adjacent pixel blocks. Moreover, all pixel blocks $(8 \times 8)$ in the image content are quantified simultaneously and independently, so that the quantization errors are not correlated. If there are quantization errors between adjacent pixel blocks, this will cause the original smooth texture to fluctuate at the boundary between adjacent blocks of pixels, that is, blocking artifacts. As shown in Figure 2, the boundary fluctuation of the reconstructed image at the receiver gives a worse visual effect to the human eye. Therefore, it is necessary to use the necessary quantization approach to reserve the original information of the image as much as possible.

4.2. Improved DCT Coefficient Quantization Approach. The monitoring of left-behind children requires low latency and clear pictures, so as to handle some exceptions in time through monitoring. There are many approaches for the reduction of blocking artifacts. As can be seen from Figure 3, there are four boundaries around pixel block 1 [28]. If the quantization error of adjacent pixel blocks on one of the boundaries is discontinuous, this will cause the blocking artifacts.

According to the spatial redundancy of the image, it can be seen that there is a little change in a certain part of the image for the pixel quantization of a pixel block with the same color, and from the characteristics of human vision, the blocking artifacts are derived from the boundary of a single frame image. When the quantization error of pixel 1 is compared with the quantization error of the other four pixel blocks, if it is smaller than the mean value of the quantization error of the other four pixel blocks, then it is not processed. On the contrary, it indicates that the boundary error of the pixel block is relatively high and the blocking artifacts may easily occur in this region. The size of $n$ of the sampling matrix (i.e., the number of nonzero values of sampling matrix) is determined by equation (6) for requantization of a pixel block so as to reduce the quantization error of the current pixel block and ensure the continuity of boundary quantization error. With the consideration of the low latency requirement of long-distance transmission, the complexity of the improved approach cannot be increased, and the source of HD video streaming is directly lost after conventional quantization compression, which leads to serious image distortion. In this paper, noise processing with zeromean noise processing is added in an optimized approach to solve the image distortion problem [29]. Given this, an improved DCT coefficient quantization approach is proposed for long-distance real-time transmission of HD video streaming.

$$
n=\left|\frac{4 \times \sigma_{1}(u, v)}{\delta \times\left(\sigma_{2}(u, v)+\sigma_{3}(u, v)+\sigma_{4}(u, v)+\sigma_{5}(u, v)\right)}\right|,
$$

where $\sigma_{1}(u, v), \sigma_{2}(u, v), \sigma_{3}(u, v), \sigma_{4}(u, v)$, and $\sigma_{5}(u, v)$ are the quantization error of each pixel block, and $\delta$ is the coefficient. 

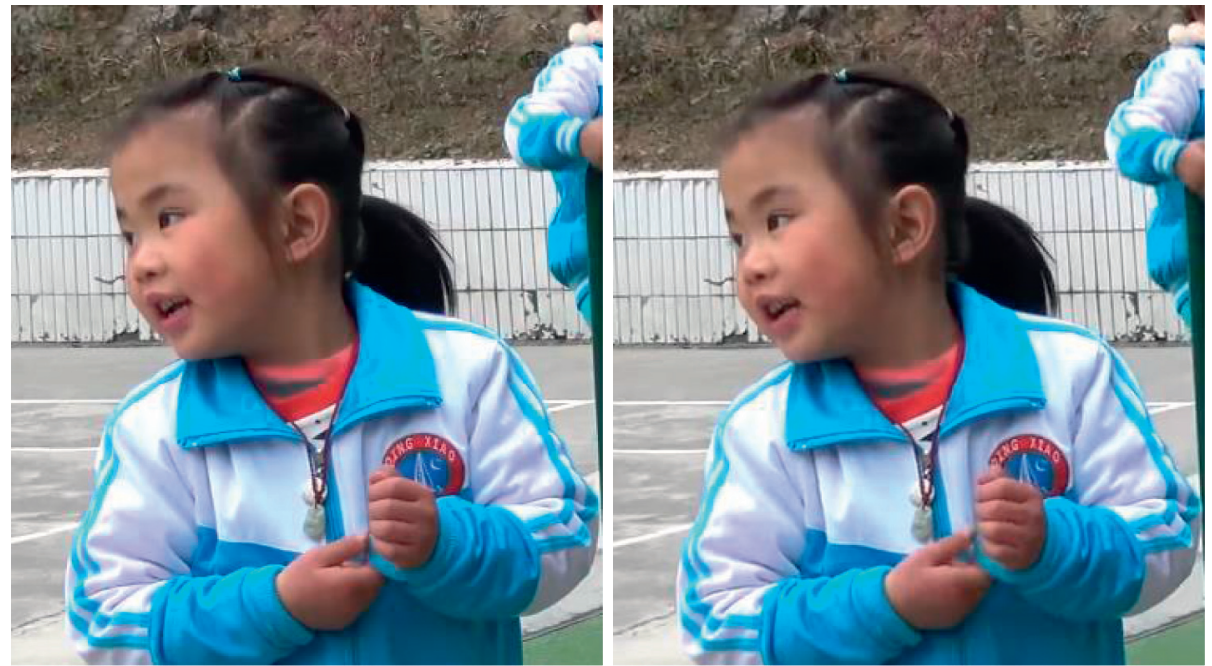

FIgURE 2: The comparison of local magnification of 2D integer DCT compression effects. (a) Original image. (b) Reconstructed image.

The optimization process is summarized as follows:

Step 1: using integer DCT and compressing DCT coefficients.

Step 2: using the boundary detection algorithm to detect the boundary of the whole reconstructed image.

Step 3: dividing the DCT coefficients into two regions (upper left and lower right), as described, most of the energy in the image is concentrated at the DCT coefficients in the upper left. Therefore, the quantization approach of DCT coefficients in the upper left is readjusted. The new quantization equations (7) and (8) are defined as follows:

$$
\begin{aligned}
& F_{Q s, \min }(u, v)=\frac{F_{D}(u, v)}{Q s(u, v)}, \\
& F_{Q s, \max }(u, v)=\frac{F_{D}(u, v)}{Q s(u, v)}+1 .
\end{aligned}
$$

Thus, each DCT coefficient in the upper left will have two possible quantized values which constitute $2^{n}$ different quantization coefficients matrix. Each quantization coefficient matrix is reconstructed, and the boundary errors of four boundaries are calculated. When the sum of squares of the four boundary error is minimized, the quantization matrix value is the quantization value of the integer DCT, which is defined as follows:

$$
\sigma^{\prime}(u, v)=\sum_{k=1}^{4}\left(F_{R}^{\prime}(u, v)-F_{D}(u, v)\right)_{k}^{2}
$$

Step 4: repeating step 3 for each pixel block at the boundary of the image. A large number of tests show that although the blocking artifacts are reduced, integer DCT belongs to unitary transformation and has the property of energy conservation. At the same time, the signal-tonoise ratio of the image may be slightly decreased after inverse DCT, and DCT coefficient value of $F(0,0), F(0,1), F(1,0), F(0,2)$, and $F(2,0)$ has a great impact on the blocking artifacts, so I select $n$ value of equation (6) no less than 5 .

The optimized DCT coefficient quantization is used to reduce the blocking artifacts of the image boundary, so that the video streaming received at the receiver and the original are consistent as much as possible, and people cannot detect the compression processing of the HD video streaming. Figure 4 shows the comparison of 2D integer DCT compression and improved quantization compression effects (the 30th frame image in the video) as the quantization step is 20. Figure 5 shows the comparison of local magnification of $2 \mathrm{D}$ integer DCT compression and improved quantization compression effects. It can be seen that the improved DCT coefficient quantization can get a better subjective visual effect.

\section{Experiment and Results Analysis}

5.1. Setup. The data source depends on the left-behind children of QingGangYuan hope primary school. QingGangYuan is a village in the city of Sinan, Guizhou Province, one of the poorest provinces in China. Among a hundred and ten students enrolled in QingGangYuan hope primary school, approximately a total of them are left-behind children or children whose parents are off to work in larger cities and rarely come home. Wireless network monitoring is used to improve the quality of leftbehind children in sports. I take the first 100 frames of monitoring video to verify the improved approach with good performance. The first 100 frames of the Foreman, Claire, Carphone, and News (QCIF format) standard video streaming sequences are encoded in intraframe, 


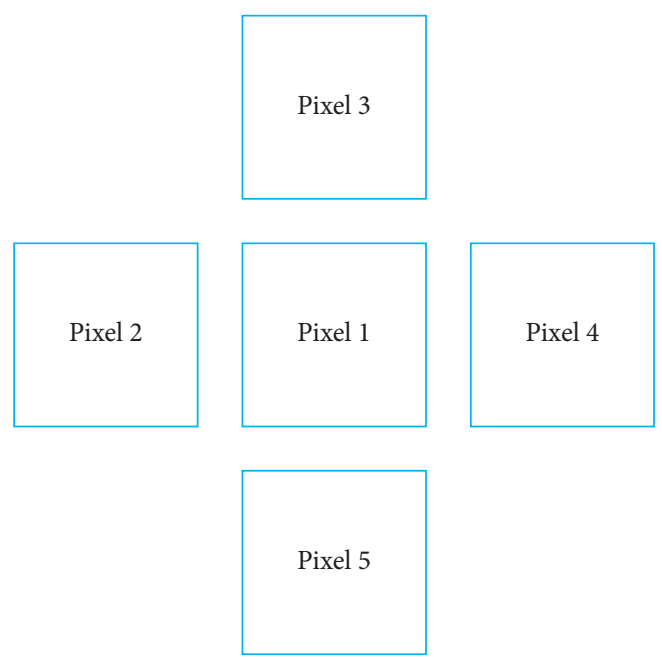

FIgURE 3: The distribution between pixel block 1 and adjacent pixel blocks.
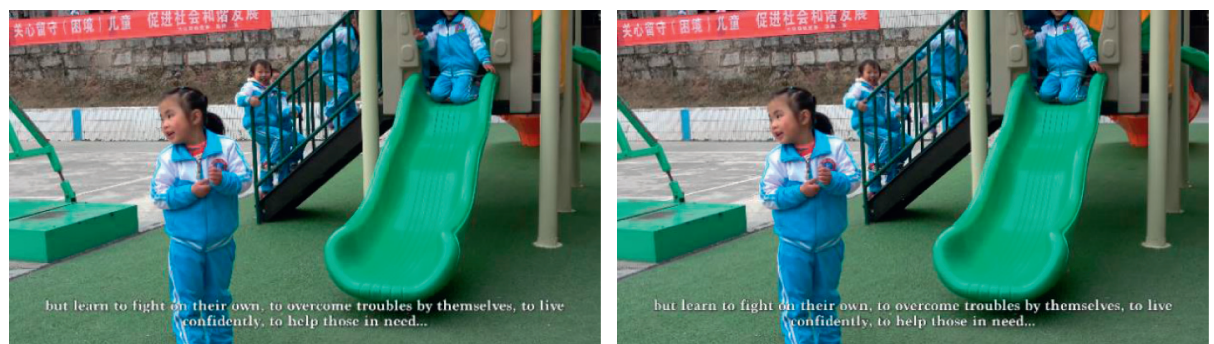

Figure 4: The comparison of 2D integer DCT compression and improved quantization compression effects. (a) Original image. (b) Reconstructed image (improved quantization).

and H.264 video encoder is used. The quantization step is 10-25. According to blocking artifacts measurement [30], the approach with improved quantization and the approach without improved quantization are for comparison in reducing the blocking artifacts.

5.2. Comparison Analysis. In Figure $6, \delta=0.9$, and Figures 6(a)-6(d) show the blocking artifact size of reconstructed images. It can be seen that the improved quantization approach can effectively reduce the blocking artifacts; in particular, when the quantization step size is large, the improved approach can be well reflected.

In Figure 7, when $\delta=0.75$ and quantization step size is 20 , the image is reconstructed at the 10th frame of the video. Among them, Figure $6(\mathrm{a})$ is the reconstructed image without the improved quantization; that is, the high-frequency information is lost through direct compression, so the reconstructed image has the worst effect. Figure 6(b) is a reconstructed image using the improved quantization approach, and the image distortion problem is solved by adding zero-mean noise processing which has greatly improved the image reconstruction effect. Figure 6(c) uses a neural network-based deblocking method to reconstruct images [31]. Figure 6(d) uses a novel frame-wise filtering method to reconstruct images [32]. The compression effect of Figure 6(a) is the worst by the naked eye, while the approach proposed in this paper seems to be the best.

Figure 8 shows the local magnification comparison of each reconstructed image in Figure 7. It can be clearly seen that the improved quantization approach has a good effect 

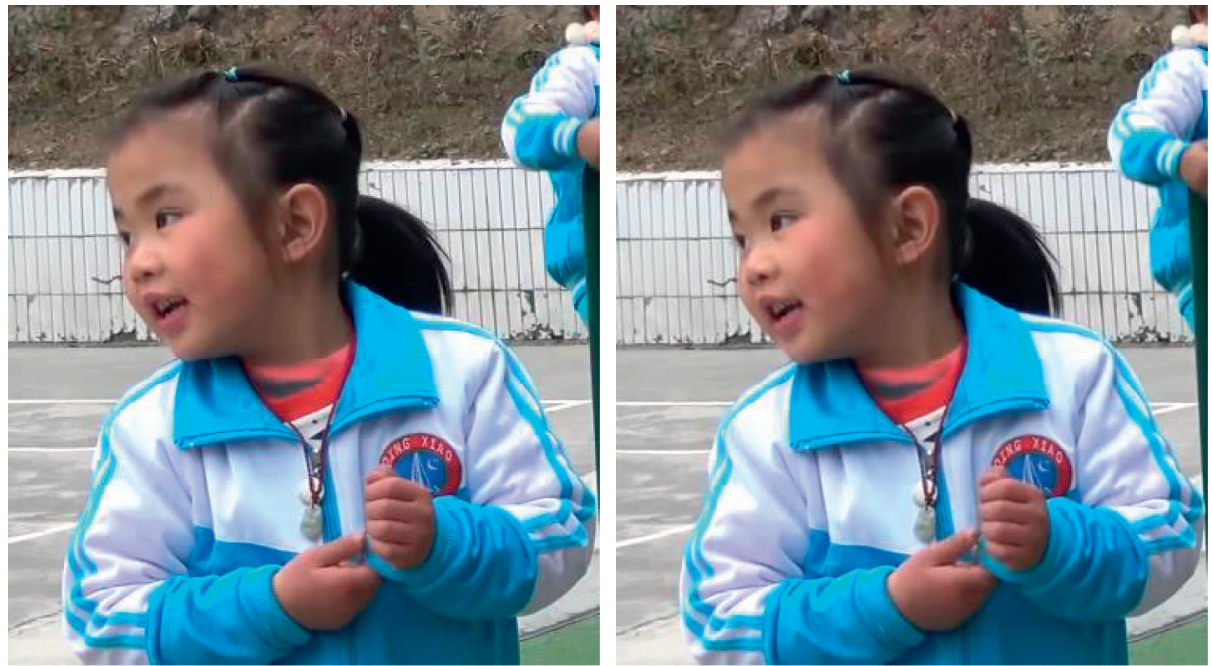

Figure 5: The comparison of local magnification of 2D integer DCT compression and improved quantization compression effects. (a) Original image. (b) Reconstructed image (improved quantization).

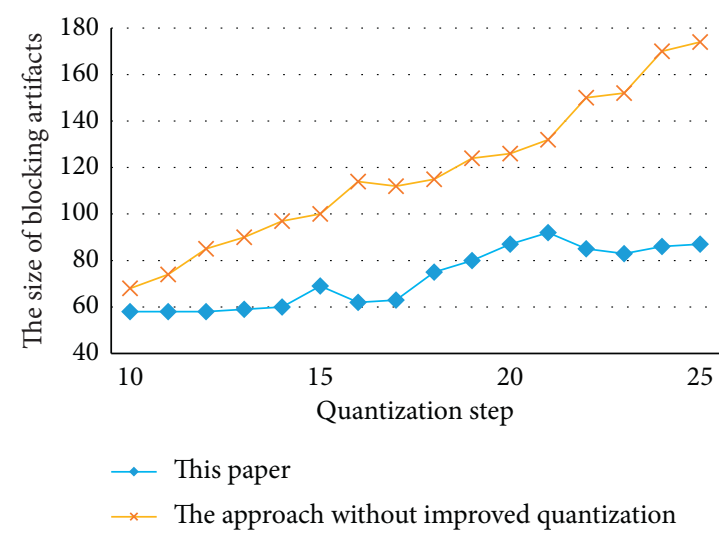

(a)

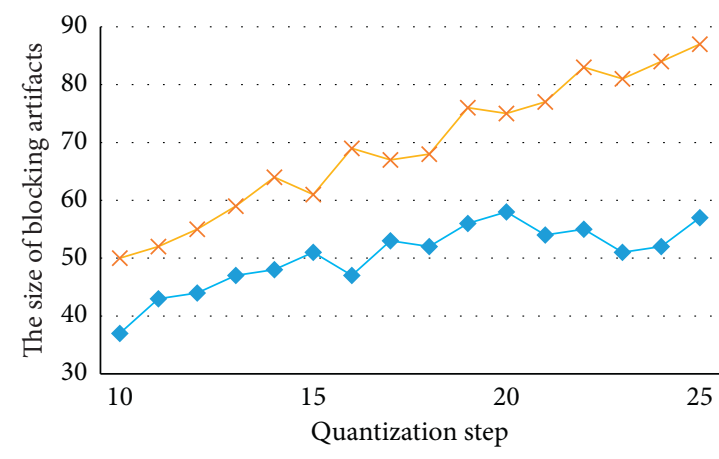

$\rightarrow$ This paper

$\rightarrow$ The approach without improved quantization

(c)

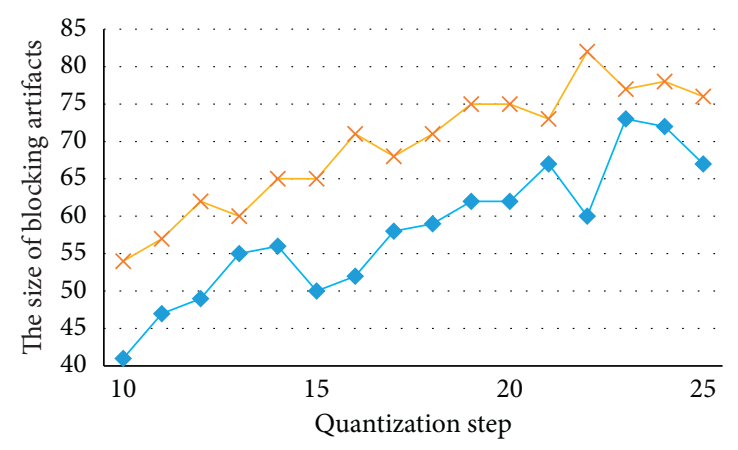

- This paper

* The approach without improved quantization

(b)

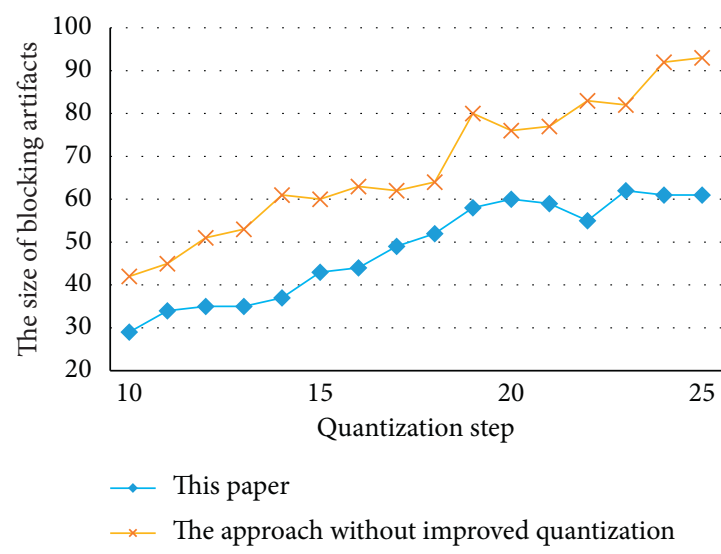

(d)

Figure 6: The comparison of reducing blocking artifacts. (a) Foreman sequence. (b) Claire sequence. (c) Carphone sequence. (d) News sequence. 


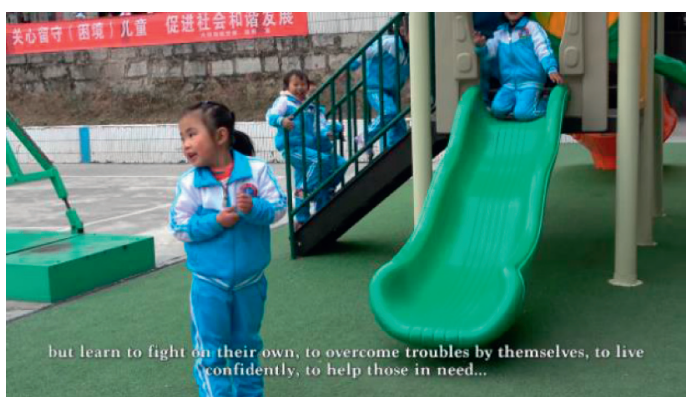

(a)

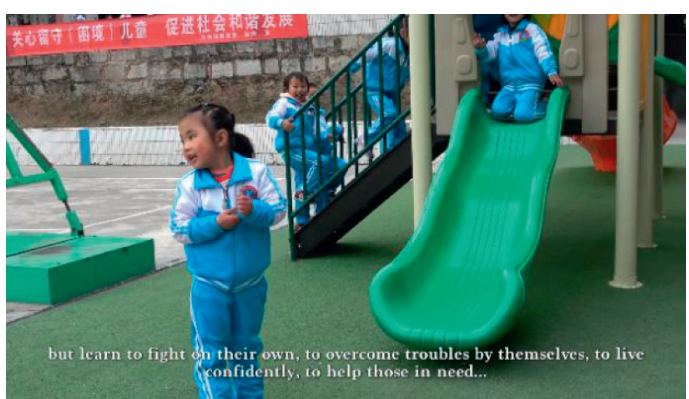

(c)

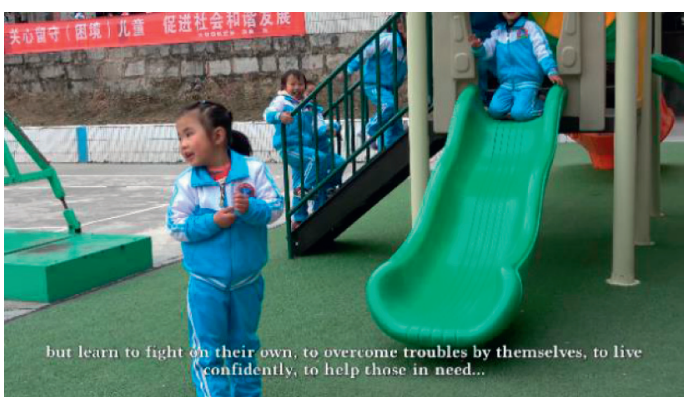

(b)

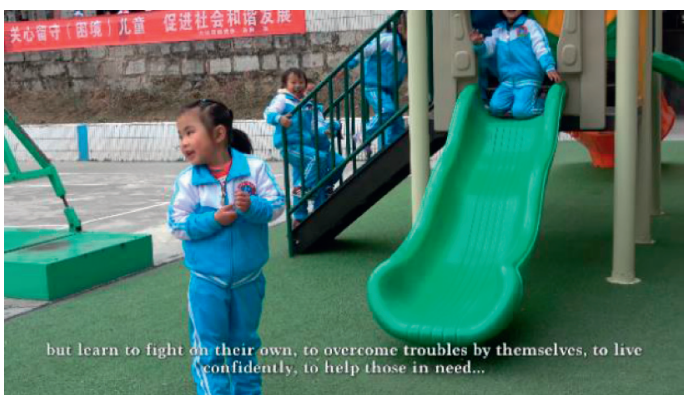

(d)

FIgURE 7: The comparison of reconstructed images at the 10th frame of the video.

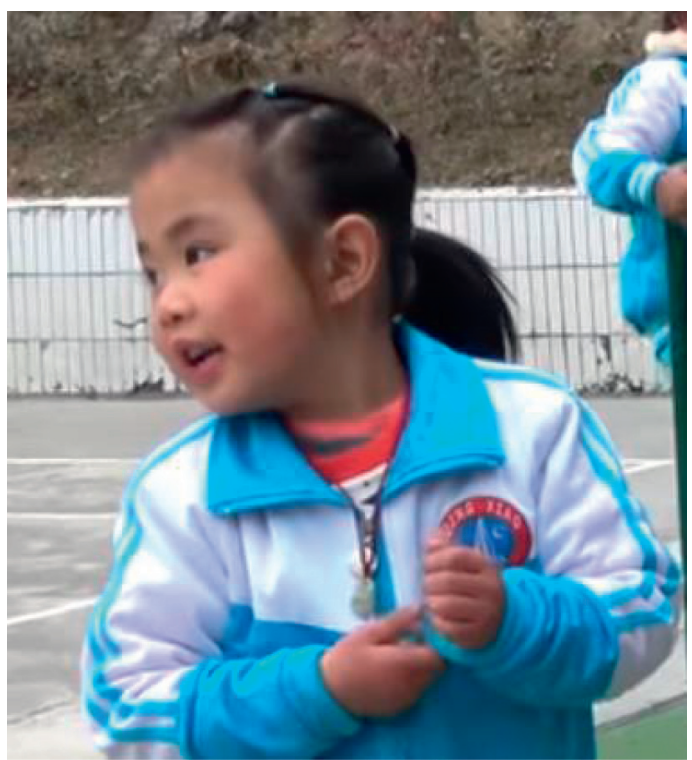

(a)

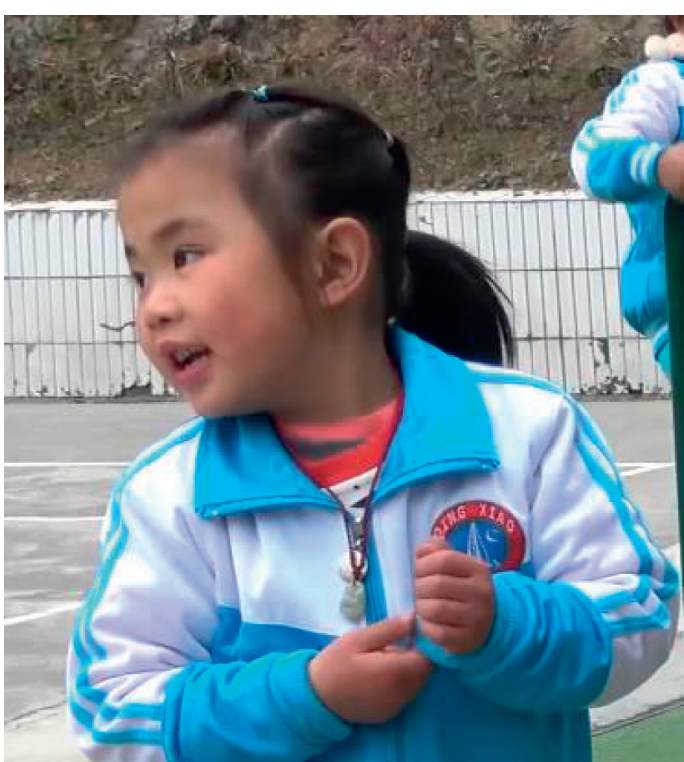

(b)

Figure 8: Continued. 


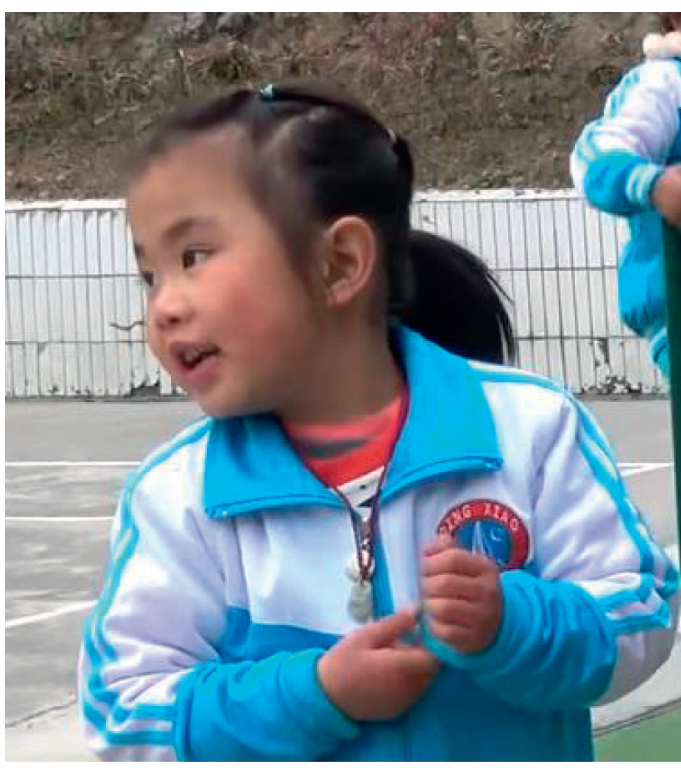

(c)

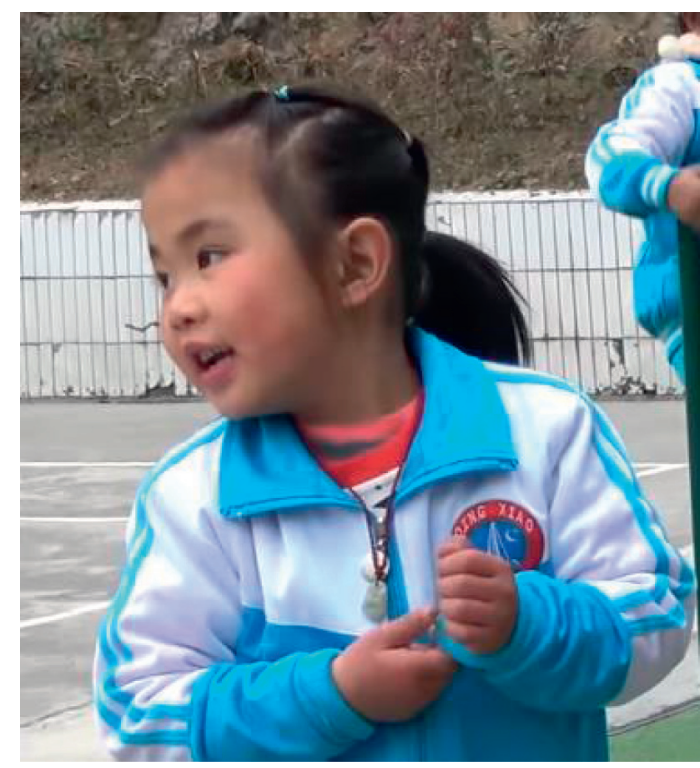

(d)

Figure 8: The comparison of local magnification of reconstructed images at the 10th frame of the video.

on the naked eye. The proposed approach can greatly reduce the blocking artifacts and improve the subjective video quality.

\section{Conclusions}

The increasing number of left-behind children in China has dramatically become a problem. They seldom take part in sports due to the lacking of monitoring. To improve the quality of leftbehind children's participation in sports based on wireless network monitoring, an improved DCT coefficient quantization approach is proposed for long-distance real-time transmission of $\mathrm{HD}$ video streaming, and the image distortion problem is solved by adding zero-mean noise processing which has greatly improved image reconstruction effect. The experimental results demonstrate that the proposed improved approach has a good performance in reducing the blocking artifacts, and when the quantization step size is large, the improved quantization approach can be well reflected. However, there are some limitations of this paper. At first, in the process of transmitting video stream over a wireless channel, the performance of the video stream is greatly disturbed by external interference, so the visual effect of the receiver needs to be improved. Then, it can get better results by using field-programmable gate array highspeed parallel processing. The approach complexity will be optimized to improve the compression efficiency for further research.

\section{Data Availability}

The data used to support the findings of this study are available from the corresponding author upon request.

\section{Conflicts of Interest}

The author declares no conflicts of interest.

\section{Acknowledgments}

This work was supported by the National Social Science Foundation: 2016 "13th Five-Year Plan" Education Youth Project, grant no. CLA160197.

\section{References}

[1] C. Bi, D. Oyserman, Y. Lin, J. Zhang, B. Chu, and H. Yang, "Left behind, not alone: feeling, function and neurophysiological markers of self-expansion among left-behind children and not left-behind peers," Social Cognitive and Affective Neuroscience, vol. 15, no. 4, pp. 467-478, 2020.

[2] J. Zhou, "Artificial intelligence driven wireless network remote monitoring based on Diffie-Hellman parameter method," Computer Communications, vol. 160, pp. 132-138, 2020.

[3] M. Taha, A. Canovas, J. Lloret, and A. Ali, "A QoE adaptive management system for high definition video streaming over wireless networks," Telecommunication Systems, vol. 77, no. 1, pp. 63-81, 2021.

[4] S. Xie, Y. Xu, Q. Shen, Z. Ma, and W. Zhang, "Modeling the perceptual quality of viewport adaptive omnidirectional video streaming," IEEE Transactions on Circuits and Systems for Video Technology, vol. 30, no. 9, pp. 3029-3042, 2020.

[5] P.-L. Chen, "A fully synthesizable ultra-N audio frequency multiplier for HDMI applications," IEEE Transactions on Circuits and Systems II: Express Briefs, vol. 67, no. 10, pp. 2134-2138, 2020.

[6] J. Chen, S. Liu, G. Deng, and S. Rahardja, "Hardware efficient integer discrete cosine transform for efficient image/video compression," IEEE Access, vol. 7, pp. 152635-152645, 2019.

[7] B. Latreche, S. Saadi, M. Kious, and A. Benziane, "A novel hybrid image fusion method based on integer lifting wavelet and discrete cosine transformer for visual sensor networks," Multimedia Tools and Applications, vol. 78, no. 8, pp. 10865-10887, 2019. 
[8] M. Zheng, J. Zheng, Z. Chen, L. Wu, X. Yang, and N. Ling, “A reconfigurable architecture for discrete cosine transform in video coding," IEEE Transactions on Circuits and Systems for Video Technology, vol. 30, no. 3, pp. 810-821, 2020.

[9] G. Paim, L. M. G. Rocha, G. M. Santana, L. B. Soares, E. A. C. da Costa, and S. Bampi, "Power-, area-, and compression-efficient eight-point approximate 2-D discrete tchebichef transform hardware design combining truncation pruning and efficient transposition buffers," IEEE Transactions on Circuits and Systems I: Regular Papers, vol. 66, no. 2, pp. 680-693, 2019.

[10] V. S. Thakur, K. Thakur, S. Gupta, and K. R. Rao, "Imageindependent optimal non-negative integer bit allocation technique for the DCT-based image transform coders," IET Image Processing, vol. 14, no. 1, pp. 11-24, 2020.

[11] D. Mukherjee and S. Mukhopadhyay, "Hardware efficient architecture for 2D DCT and IDCT using Taylor-series expansion of trigonometric functions," IEEE Transactions on Circuits and Systems for Video Technology, vol. 30, no. 8, pp. 2723-2735, 2020.

[12] N. Brahimi, T. Bouden, T. Brahimi, and L. Boubchir, "A novel and efficient 8-point DCT approximation for image compression," Multimedia Tools and Applications, vol. 79, no. 1112, pp. 7615-7631, 2020.

[13] V. Arunachalam, A. N. Joseph Raj, and S. Deepika, "Performance improvement of vector-radix decimation-in-frequency 3D-DCT/IDCT using variable word length," Circuits, Systems, and Signal Processing, vol. 40, no. 4, pp. 1818-1831, 2021.

[14] A. Singhadia, P. Bante, and I. Chakrabarti, "A novel algorithmic approach for efficient realization of 2-D-DCT architecture for HEVC," IEEE Transactions on Consumer Electronics, vol. 65, no. 3, pp. 264-273, 2019.

[15] N. Sivasubramanian and G. Konganathan, "A novel semi fragile watermarking technique for tamper detection and recovery using IWT and DCT," Computing, vol. 102, no. 6, pp. 1365-1384, 2020.

[16] S. Chatterjee and K. Sarawadekar, "WHT and matrix decomposition-based approximated IDCT architecture for HEVC," IEEE Transactions on Circuits and Systems II: Express Briefs, vol. 66, no. 6, pp. 1043-1047, 2019.

[17] R. Sinhal, D. K. Jain, and I. A. Ansari, "Machine learning based blind color image watermarking scheme for copyright protection?" Pattern Recognition Letters, vol. 145, pp. 171-177, 2021.

[18] C.-H. Yeh, C.-H. Lin, M.-H. Lin, L.-W. Kang, C.-H. Huang, and M.-J. Chen, "Deep learning-based compressed image artifacts reduction based on multi-scale image fusion," Information Fusion, vol. 67, pp. 195-207, 2021.

[19] J. Li, D. Li, C. Chen, Q. Yan, and X. Lu, "A dual-residual network for JPEG compression artifacts reduction," Signal, Image and Video Processing, vol. 15, no. 3, pp. 485-491, 2021.

[20] A. Singh and J. Singh, "A content adaptive method of deblocking and super-resolution of compressed images," Multimedia Tools and Applications, vol. 80, no. 7, pp. 1109511131, 2021.

[21] M.-T. Wu, "Wavelet transform based on Meyer algorithm for image edge and blocking artifact reduction," Information Sciences, vol. 474, pp. 125-135, 2019.

[22] N.-U. Kim and Y.-L. Lee, "Blocking-artifact detection in frequency domain for frame-rate up-conversion," Personal and Ubiquitous Computing, vol. 22, no. 1, pp. 173-184, 2018.

[23] Z. Bahaoui, K. Zenkouar, H. E. Fadili, H. Qjidaa, and A. Zarghili, "Blocking artifact removal using partial overlapping based on exact Legendre moments computation," Journal of Real-Time Image Processing, vol. 14, no. 2, pp. 433-451, 2018.

[24] K. Al-Khayyat, I. Al-Shaikhli, and M. Al-Hagery, "Second compression for pixelated images under edge-based compression algorithms: JPEG-LS as an example," Journal of Intelligent and Fuzzy Systems, vol. 40, no. 6, pp. 10661-10669, 2021.

[25] S. Kudo, S. Orihashi, R. Tanida, S. Takamura, and H. Kimata, "GAN-based image compression using mutual information for optimizing subjective image similarity," IEICE Transactions on Information and Systems, vol. E104.D, no. 3, pp. 450-460, 2021.

[26] A. M. Sandu, C. A. Mifiale, M. A. A. Ungureanu, and E. I. Scarlat, "Case comparison between direct image compression and hologram compression," University Politehnica of Bucharest Scientific Bulletin-Series A-Applied Mathematics and Physics, vol. 83, no. 1, pp. 235-246, 2021.

[27] D. Tellez, G. Litjens, J. van der Laak, and F. Ciompi, "Neural image compression for gigapixel histopathology image analysis," IEEE Transactions on Pattern Analysis and Machine Intelligence, vol. 43, no. 2, pp. 567-578, 2021.

[28] H. Zhang, B. Zheng, and H. Zu, "Application of SVGA video real-time transmission technology in music education information communication," Journal of Intelligent and Fuzzy Systems, vol. 39, no. 2, pp. 1733-1744, 2020.

[29] X. Xue and J. Zhang, "Matching large-scale biomedical ontologies with central concept based partitioning algorithm and adaptive compact evolutionary algorithm," Applied Soft Computing, vol. 106, pp. 1-11, 2021.

[30] P. He, X. Jiang, T. Sun, and S. Wang, "Detection of double compression in MPEG-4 videos based on block artifact measurement," Neurocomputing, vol. 228, pp. 84-96, 2017.

[31] Y. Zhang, E. Salari, and S. Zhang, "Reducing blocking artifacts in JPEG-compressed images using an adaptive neural network-based algorithm," Neural Computing \& Applications, vol. 22, no. 1, pp. 3-10, 2013.

[32] H. Huang, I. Schiopu, and A. Munteanu, "Frame-wise CNNbased filtering for intra-frame quality enhancement of HEVC Videos," IEEE Transactions on Circuits and Systems for Video Technology, vol. 31, no. 6, pp. 2100-2113, 2021. 